\title{
Computational Knee Ligament Modeling Using Experimentally Determined Zero-Load Lengths
}

\author{
Katherine H. Bloemker ${ }^{1} *$, Trent M. Guess ${ }^{1}$, Lorin Maletsky ${ }^{2}$, Kevin Dodd ${ }^{2}$ \\ ${ }^{I}$ Musculoskeletal Biomechanics Research Lab, Department of Civil and Mechanical Engineering, University of Missouri \\ - Kansas City, Kansas City, MO \\ ${ }^{2}$ Department of Mechanical Engineering, University of Kansas, Lawrence, $K S$
}

\begin{abstract}
This study presents a subject-specific method of determining the zero-load lengths of the cruciate and collateral ligaments in computational knee modeling. Three cadaver knees were tested in a dynamic knee simulator. The cadaver knees also underwent manual envelope of motion testing to find their passive range of motion in order to determine the zero-load lengths for each ligament bundle. Computational multibody knee models were created for each knee and model kinematics were compared to experimental kinematics for a simulated walk cycle. One-dimensional non-linear spring damper elements were used to represent cruciate and collateral ligament bundles in the knee models. This study found that knee kinematics were highly sensitive to altering of the zero-load length. The results also suggest optimal methods for defining each of the ligament bundle zero-load lengths, regardless of the subject. These results verify the importance of the zero-load length when modeling the knee joint and verify that manual envelope of motion measurements can be used to determine the passive range of motion of the knee joint. It is also believed that the method described here for determining zero-load length can be used for in vitro or in vivo subject-specific computational models.
\end{abstract}

Keywords: Computational knee modeling, ligament parameters, reference strain, zero-load length.

\section{INTRODUCTION}

Computational modeling of the knee helps us better understand the forces and strains placed on knee structures, such as the ligaments, during ambulatory activities. With better understanding of ligament strains during different loading situations, we are able to more accurately determine the cause of ligament injury. In addition, computational knee models bring insight for rehabilitation and surgical ligament repair [1]. Weiss et al. acknowledged that "verification and validation" of computational models is necessary to more accurately define ligament function in the knee [2]. This study uses a previously validated computational platform to study the method of determining the zero-load length used in the one-dimensional discrete element representation of ligaments.

Various methods have been used to represent ligaments in computational models including finite element techniques and elastic springs [3]. Modeling the ligaments as elastic springs is the most computationally efficient method and several studies have looked at how these elastic springs should be defined. Some have defined the springs as completely linear [1] while others used non-linear springs to represent ligament buckling under compression and the toe region of the ligaments [3-10]. Studies have shown that ligaments have a non-linear toe region which occurs because of the initial crimping of the ligament fibers and this toe

*Address correspondence to this author at the 370B Robert H. Flarsheim Hall 5100 Rockhill Road Kansas City, Missouri 64110; Tel: 816-235-5639; Fax: 816-235-1260; E-mail: bloemkerk@umkc.edu region ends when all of the fibers have become taut [2]. At that point the ligaments behave as a linear spring with a stiffness parameter, $\mathrm{k}$. This study uses a non-linear onedimensional spring method to define the cruciate and collateral ligaments of the knee.

The method for defining each ligament used in this study is the force-displacement curve that was first introduced by Wismans et al. [11] and Blankevoort et al. [5]. Many other studies have used this method [4-12]. This study uses the ligament modeling terminology used by Blankevoort et al. [5]. The zero-load length $\left(l_{0}\right)$ is defined as the length of the ligament when it first becomes taut. The reference length $\left(l_{r}\right)$ is the length of the ligament at the reference (typically extension) position and the reference strain $\left(\varepsilon_{r}\right)$ is the strain in each ligament at that reference position [5]. Previous studies using this force-displacement curve find the zeroload length by using the reference length and previously published reference strain values [5-7, 12]. This method, which will be called the reference strain method in this paper, uses these generalized reference strain values which do not take subject specific ligament information into account. The reference strain method is an easy approach to modeling the ligaments since it is difficult to find data for the actual zero-load length of knee ligaments [7]. Due to this, the zero-load lengths in other previous knee models have been inferred through an optimization process comparing experimental kinematics to model kinematics $[7,9]$. In this method, cadaver knees are loaded with known forces (for example with a robotic testing system [13]) and ligament parameters are modified until the kinematic error between model and experiment is minimized. This method has proven 
Table 1. Information Regarding Each Cadaver Knee Used in this Study

\begin{tabular}{|l|c|c|c|c|c|}
\hline & Age at death & Gender & Right or Left & Height (in) & Weight (lbs) \\
\hline \hline Knee \#1 & 77 & Male & Right & 70 & 220 \\
\hline Knee \#2 & 55 & Female & Left & 67 & 160 \\
\hline Knee \#3 & 78 & Female & Right & 65 & 130 \\
\hline
\end{tabular}

to be valid for modeling cadaver knee ligaments, but may not translate to in vivo subjects very well. In the current study the zero-load length was experimentally determined by measuring the passive limits of the knee joint and then calculating the extent of motion for each ligament bundle. A correction percentage (which will be called the zero-load length percentage in this paper) was then applied to the extent of motion for each ligament to determine its zero-load length.

A first objective of this study was to determine the sensitivity of the kinematics of the knee joint to the zero-load length percentage. Weiss et al. [2] and Baldwin et al. [4] "advocate performing sensitivity studies, especially when applying population averages to subject-specific models." Sensitivities of many of the parameters in the ligament equations have not been evaluated, especially the zero-load length percentage, although a few sensitivity studies have been performed. Bertozzi et al. [1, 14] studied the length reached by each ligament bundle during passive flexion and the elastic modulus of the cruciates. Baldwin et al. [4] studied the ligament stiffness, reference strain, and attachment site locations for the cruciates and collaterals. The present study compares three separate cadaver knees where the previous studies only investigated one knee.

The main goal of this study was to develop a method to determine key ligament parameters in vitro and possibly in the future, in vivo. It is believed that the approach presented in this paper to finding subject specific zero-load lengths will translate easily to in vivo subjects since it is based on the laxity of the knee joint. Another goal of this study was to find values for this zero load-length percentage that can be widely used, regardless of the subject. The final goal of this study was to compare the kinematic results of this subject specific method as compared to the generic reference strain method.

\section{METHODS}

\section{Cadaver Knee Measurements and Testing}

Three cadaver knees were used for this ligament study (Table 1). The three knees were fresh frozen until testing and each underwent magnetic resonance imaging (using a Siemens 1.5 $\mathrm{T}$ machine with knee coil) after thawing. The program 3D Slicer (www.slicer.org) was used to create the bone, cartilage, and ligament geometries from the magnetic resonance images using manual segmentation. Geomagic Studio (Geomagic, Inc. Research Triangle Park, NC) was used for file conversion and post-process filtering of the knee geometries, which included smoothing, removing spikes, and reducing noise.

After imaging, each cadaver knee was mounted in a dynamic knee simulator (Kansas Knee Simulator, University of Kansas, Lawrence, KS). The femur and tibia were mounted in the dynamic knee simulator by potting the bones into fixtures using bone cement. The knee simulator replicates the loading and motion during activities, such as walking, using servo-hydraulic actuators which control the machine using five axes of control (quadriceps force, vertical force applied at the hip, medial-lateral ankle force, ankle vertical torque, and ankle flexion torque) [15]. Loading profiles for the machine were generated from a previously validated computational model and the position and force at each axis were measured during simulation [16]. Each knee underwent multiple walk cycle simulations. The walk cycle was created to simulate the ISO standard for knee wear simulations [17]. For the walk cycle, the simulated quadriceps muscle controlled the flexion angle of the femur at the hip and the other four actuators applied dynamic loading at the hip and ankle. Kinematics of the femur, tibia, and patella were obtained using rigid body markers and a 3camera Optotrak 3020 system (Northern Digital Inc., Waterloo, Ontario). This system has a measurement bias of $0.05^{\circ}$ and a $95 \%$ repeatability limit of $0.67^{\circ}$ for a rotation of $10^{\circ}$, and a bias of $0.03 \mathrm{~mm}$ and a $95 \%$ repeatability limit of $0.29 \mathrm{~mm}$ for a translation of $10 \mathrm{~mm} \mathrm{[18]}$. The position and orientation of each cadaver femur, tibia, and patella relative to the dynamic knee simulator were recorded using a probing tip with the Optotrak system. Collected points included point clouds of bone and cartilage surfaces, ligament insertion locations, and reference points on the dynamic knee simulator.

\section{Multibody Knee Models}

The method for developing the multibody knee models was the same as that described by Guess et al. [8]. The knee geometries (tibia, femur, patella, and articular cartilage) were placed into a validated multibody model of the dynamic knee simulator developed in MD Adams (MSC Software Corporation, Santa Ana, CA) (Fig. 1). The anatomical points identified during the cadaver testing were used to align the knee geometries (tibia, femur, and patella) with the machine in the computational model. Compliant contacts were then defined between the articulating surfaces of the tibia, patella, and femur geometries. The compliant contact used was the default MD Adams contact defined as:

$$
F=k \delta^{\exp }+B \dot{\delta}
$$

where $\mathrm{F}$ is the contact force, $\delta$ is the interpenetration of the two geometries, $\mathrm{k}$ is the spring constant, and $\mathrm{B}$ is the damping coefficient. The values used in this study were: $\mathrm{k}=$ $500 \mathrm{~N} / \mathrm{mm}^{1.5}, \exp =1.5$, and $\mathrm{B}=5 \mathrm{Ns} / \mathrm{mm}[8]$.

The patellar tendon was represented by three bundles and the quadriceps tendons were represented with four bundles. The patellar and quadriceps tendon force-length relationships were based on literature [19]. None of the three models in this study included the meniscus. 


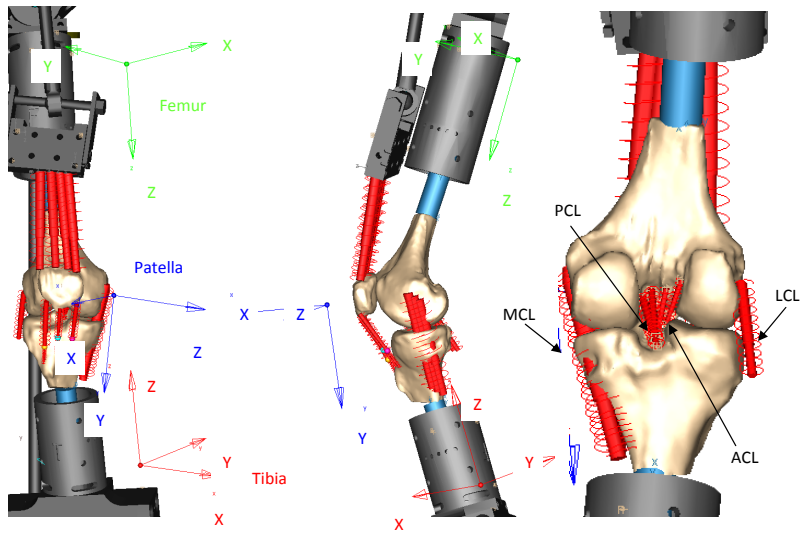

Fig. (1). Three views of the Knee \#1 model in the knee simulator. Also shown are the femur, tibia, and patella coordinate systems. The $\mathrm{z}$-axis of the femur coordinate is approximately aligned with the long axis of the femur. The y-axis of the femur coordinate is primarily in the anterior-posterior direction while the $\mathrm{x}$-axis is primarily oriented in the medial-lateral direction. Also shown is the posterior view with each of the ligament bundles labeled.

\section{Ligament Properties}

The anterior cruciate ligament (ACL) and the posterior cruciate ligament (PCL) were modeled as two bundles each aACL, pACL and aPCL, pPCL $[20,21]$. The lateral collateral ligament (LCL) and the medial collateral ligament (MCL) were modeled as three bundles each - aLCL, sLCL, pLCL and aMCL, iMCL, pMCL [22] (Fig. 1). Onedimensional non-linear spring damper elements were used for both the cruciate and the collateral ligaments in the multibody models. The ligament stiffness parameters (k), shown in Table 2, are taken from the literature [5, 11]. A damping coefficient of $0.5 \mathrm{Ns} / \mathrm{mm}$ in parallel with the spring element was used for each ligament bundle [8]. Non-linear equations which take the toe region into account were used for modeling each ligament bundle in MD Adams. These equations were derived from the ligament force as a function of strain, the length of each ligament in the position it was constructed in MD Adams, and the measured zero-load length. The length of the ligament in the position it was constructed in MD Adams is used as the original ligament length therefore ligament displacement is derived from that starting point. The force-displacement curve for the ligaments is described by equations (2) and (3) $[5,11]$ where $\mathrm{k}$ is the stiffness parameter, $l_{0}$ is the zero-load length, and $\varepsilon_{l}$ is the spring parameter assumed to be 0.03 [23].

$$
\begin{aligned}
& f= \begin{cases}\frac{1}{4} k \varepsilon^{2} / \varepsilon_{l} & 0 \leq \varepsilon \leq 2 \varepsilon_{l} \\
k\left(\varepsilon-\varepsilon_{l}\right) & \varepsilon>2 \varepsilon_{l} \\
0 & \varepsilon<0\end{cases} \\
& \varepsilon=\left(\frac{l-l_{0}}{l_{0}}\right)
\end{aligned}
$$

$l_{0}=\left(\frac{l_{r}}{\varepsilon_{r}+1}\right)$

The zero-load length $\left(l_{0}\right)$ is defined as the length of the ligament when it first becomes taut. This should not be confused with the reference length $\left(l_{r}\right)$ which is shown in equation (4). The reference length is the length of the ligament at the reference or extension position and the reference strain $\left(\varepsilon_{r}\right)$ is the strain in each ligament at that reference position [5]. Most previous studies using this force-displacement curve find the zero-load length by using equation (4). This will be called the reference strain method in this paper. This study does not use equation (4), but rather finds the zero-load lengths of the ligament bundles experimentally.

Subject specific ligament insertion and origin sites and subject specific zero-load lengths were used for each bundle [10]. Insertions and origins for the ligaments were determined by dissection and probing the cadaver knees after testing was completed. The probing tip with the Optotrak system was used to measure points surrounding the attachment sites for each ligament on the tibia and femur. The specific discrete insertion site for each 1-D bundle within the total ligament insertion area was determined from studies available in the literature [20, 24-28]. The method used to probe anatomical points manually using the Optotrack system on a disarticulated cadaver knee has been shown to be repeatable and accurate. Morton et al. [29] had multiple orthopedic surgeons, research professionals, and research assistants probe disarticulated cadaver knee geometries for specific anatomical landmarks and found standard deviations of less than $2.2 \mathrm{~mm}$ when measuring the lateral tibial condyle.

The kinematic envelope of motion (KEM) is the range the knee can move while minimally straining the ligaments (while applying minimal force). The KEM was measured by manually manipulating the knee throughout the flexion range until some restraint was felt by the researcher when moving in different directions [30]. The zero-load length of each ligament was determined by calculating the maximum straight-line distance between insertion and origin sites throughout the entire motion for each ligament bundle and then applying a correction percentage (the zero-load length percentage). To determine the KEM of the tibio-femoral joint the femur was held fixed in a horizontal position while the tibia was manually moved through its full range of motion. Care was taken to apply minimal force to the tibia while it was being manipulated. The rigid-body motion of the tibia relative to the femur was recorded by the Optotrak system during this process.

The KEM measured the motion of the tibia relative to the femur through its full range of laxity motion. The previously determined tibial insertion site for each ligament bundle was represented in the local tibia coordinate system. Similarly, the femoral insertion site for each ligament bundle was

Table 2. Stiffness Parameters used for Each Ligament Bundle in this Study (in Terms of Force Versus Strain)

\begin{tabular}{|c|c|c|c|c|}
\hline & aACL and pACL & aPCL and pPCL & aLCL, sLCL, pLCL & aMCL, iMCL, pMCL \\
\hline \hline $\mathrm{k}[\mathrm{N}]$ & 5000 & 9000 & 2000 & 2750 \\
\hline
\end{tabular}




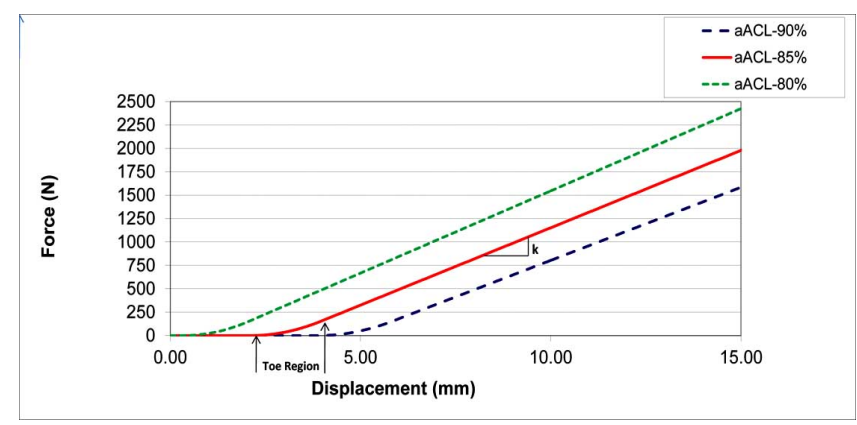

Fig. (2). Force-displacement curves for the aACL for Knee \#1 at all five zero-load length percentages $(75 \%, 80 \%, 85 \%, 90 \%$, and $95 \%)$. The non-linear toe region and linear stiffness $(\mathrm{k})$ are pointed out on the $85 \%$ curve. This figure shows how changing the zeroload length percentage impacts the non-linear toe region. Note that linear stiffness $(\mathrm{k})$ in this figure has the units $\mathrm{N} / \mathrm{mm}$.

represented in the local femoral coordinate system. The maximum distance between the tibial insertion and femoral insertion was then calculated for the KEM of each knee. The actual zero-load length was obtained by taking a percentage (zero-load length percentage) of the maximum distance calculated for each ligament bundle during the passive KEM. A percentage of $100 \%$ would mean that the distance measured between the tibial and femoral insertions during the manual manipulation is the actual zero-load length of the ligament. However, it is difficult to manually manipulate the knee and apply no force across the joint and ligaments which is why the percentage is applied. Ligaments that were stretched much further than the actual zero-load length will require a much lower correction percentage than those that were not stretched as far. Previous studies of this type estimated the zero-load length percentage to be $85 \%$ for all of the ligament bundles [8]. To date, this parameter has not been fully investigated. In addition, the zero-load length percentage used in previous studies has always been uniform for all of the ligaments (cruciates and collaterals).

\section{Zero-Load Length Percentage}

In this study, the cruciates (ACL and PCL) were treated similarly as well as the collaterals (LCL and MCL). The zero-load length percentages were then systematically changed for each set of ligament bundles. Zero-load length percentages for this study ranged in 5\% increments from $75 \%$ to $95 \%$. The non-linear toe region varies depending on the zero-load length percentage used (Fig. 2). The point of zero displacement in Fig. (2) correlates to the length of the ligament in the position it was constructed in the MD Adams model. The simulation parameters are shown in Table 3 .

\section{Multibody Knee Model Walk Cycle Simulations}

The method used for knee model simulations was the same as that described by Guess et al. [8]. The multibody knee models were validated by simulating the same walk cycle on the computational models as was performed on the cadaver knees in the dynamic knee simulator. Bone kinematics were then compared between the computational model and the cadaver knee. The walk cycle was based on ISO specification 14243-1:2002 [17] and the actuators in the dynamic knee simulator were controlled to reproduce both the loading and motion of the walk cycle on each cadaver knee. For one gait cycle $(100 \%)$, heel strike occurred at
Table 3. Simulation Parameters Used in the Zero-Load Length Percentage Study

\begin{tabular}{|c|c|c|}
\hline & $\begin{array}{c}\text { Cruciates } \\
\text { (ACL and PCL) }\end{array}$ & $\begin{array}{c}\text { Collaterals } \\
\text { (LCL and MCL) }\end{array}$ \\
\hline \hline 1 & $95 \%$ & $95 \%$ \\
\hline 2 & $90 \%$ & $90 \%$ \\
\hline 3 & $85 \%$ & $85 \%$ \\
\hline 4 & $80 \%$ & $80 \%$ \\
\hline 5 & $75 \%$ & $75 \%$ \\
\hline 6 & $95 \%$ & $75 \%$ \\
\hline 7 & $75 \%$ & $95 \%$ \\
\hline 8 & $90 \%$ & $80 \%$ \\
\hline 9 & $80 \%$ & $90 \%$ \\
\hline 10 & $95 \%$ & $85 \%$ \\
\hline 11 & $85 \%$ & $95 \%$ \\
\hline 12 & $85 \%$ & $75 \%$ \\
\hline 13 & $75 \%$ & $85 \%$ \\
\hline 14 & $85 \%$ & $80 \%$ \\
\hline 15 & $85 \%$ & $90 \%$ \\
\hline
\end{tabular}

approximately $0 \%$, mid stance occurred at approximately $20 \%$, and toe-off occurred at approximately 60\%. Due to limitations of the dynamic knee simulator and to protect the cadaver knees, each walk cycle was modified to last 10 seconds.

A feedback control loop in the computational models adjusted the quadriceps force in order to maintain the desired hip flexion angle (note: hip flexion angle was measured during the cadaver testing and was used as an input to the computational model simulations). The other simulation inputs were the vertical load at the hip and the three loads applied at the ankle. In the model these loads were applied as force vectors.

The measured kinematics included the relative position and orientation of the tibia and patella coordinate systems relative to the femur coordinate system (Fig. 1). Coordinate systems are based on the physical location of the rigid body markers that were used for the Optotrak system during experimental testing. This coordinate system was not transformed to anatomical coordinates because comparisons were made directly between the experimental data and the model results. The orientation measurements taken were body 1, 2, and 3 orientation angles (1,2,3 Euler angle sequence) of the tibia relative to the femur. The translation measurements taken were $\mathrm{x}, \mathrm{y}$, and $\mathrm{z}$ translation of the tibia relative to the femur represented in femoral coordinates. Walk cycles were run for each of the three knee models with each of the previously explained zero-load length percentages.

In addition, the method described by Blankevoort et al. [5] in which the zero-load length $\left(l_{0}\right)$ is found from equation (4) using previously published reference strain values $\left(\varepsilon_{r}\right)$ was implemented on each of the three knee models. In this reference strain method, the reference length $\left(l_{r}\right)$ was 
Table 4. The Average Translation (x,y,z) RMS Errors and the Normalized Translation Errors for the Tibia Coordinate System Relative to the Femoral Coordinate System for Each Knee During each Simulation. Normalized Error Values for Each Simulation were Added for all Three Knees. Note: The Top Three Values in each Column are Shown in Bold

\begin{tabular}{|c|c|c|c|c|c|c|c|}
\hline Tibia xyz & \multicolumn{2}{|c|}{ Knee \#1 } & \multicolumn{2}{|c|}{ Knee \#2 } & \multicolumn{2}{|c|}{ Knee \#3 } & Norm Sum \\
\hline 1 & 7.25 & 0.062 & 6.42 & 0.187 & N/A & N/A & N/A \\
\hline 2 & 7.31 & 0.069 & 5.69 & 0.123 & 45.26 & 1 & 1.19 \\
\hline 3 & 8.77 & 0.263 & 5.30 & 0.890 & 18.73 & 0.357 & 0.710 \\
\hline 6 & 7.61 & 0.110 & 5.36 & 0.095 & N/A & N/A & N/A \\
\hline 7 & 8.94 & 0.286 & 15.72 & 1 & 34.96 & 0.750 & 2.04 \\
\hline 8 & 7.84 & 0.140 & 4.28 & 0 & N/A & N/A & N/A \\
\hline 9 & 7.65 & 0.115 & 8.85 & 0.400 & 31.12 & 0.657 & 1.17 \\
\hline 12 & 7.89 & 0.147 & 4.29 & 0.001 & 5.53 & $\mathbf{0 . 0 3 7}$ & 0.185 \\
\hline 13 & 14.31 & 1 & 8.93 & 0.406 & 13.77 & 0.237 & 1.64 \\
\hline 14 & 8.04 & 0.166 & 4.57 & 0.025 & 8.92 & 0.119 & 0.311 \\
\hline 15 & 7.58 & 0.106 & 6.79 & 0.193 & 26.96 & 0.556 & 0.855 \\
\hline
\end{tabular}

measured for each ligament bundle when the knee models were in full extension.

For each simulation, RMS errors between predicted kinematics and experimentally measured kinematics for the tibia were calculated. The RMS values used for the comparison were three translation errors in millimeters $(\mathrm{x}, \mathrm{y}$, z) and three orientation errors in degrees (Body 1, 2, 3). The average of the translation RMS errors and the average of orientation RMS errors were calculated for each simulation and then each was compared.

The average RMS errors for each simulation were normalized for each knee (a value of 0 corresponds to the lowest RMS error average for that particular knee, or the most accurate simulation). The normalized error values for each knee were then added together to find the best overall simulations for all of the knees combined. Normalization was done by using the following equation:

Norm_Error $=\frac{A v g-\operatorname{Min}}{\operatorname{Max}-\operatorname{Min}}$

Analysis of variance (ANOVA) was also performed on the averaged RMS error results to determine the significant factors $(\alpha=0.05)$. The two factors that were tested were the zero-load length percentage for ACL/PCL and LCL/MCL.

\section{RESULTS}

Table 4 shows the average RMS errors for tibia translation for each knee. Table $\mathbf{5}$ shows the average RMS errors for tibia orientation for each knee. Tibia normalized error results for zero-load length percentage are also shown in Tables 4 and 5. It should be noted that for Knee \#3 the femur slipped off of the tibial plateau at certain levels (when the cruciates were too lax, at $90 \%$ and $95 \%$ ). This resulted in failed walk cycles for the following simulations: (1) All 95\%, (6) ACL/PCL 95\% LCL/MCL 75\%, (8) ACL/PCL $90 \%$ LCL/MCL $80 \%$, and the reference strain method. The overall best simulation turned out to be (12) ACL/PCL $85 \%$ and LCL/MCL 75\%. The specific RMS results for each knee for this simulation are shown in Table 6. Fig. (3) shows $100 \%$ gait cycle comparing tibia position and orientation for experimental vs. model for Knee \#1 at this best case scenario. Table 6 also shows the RMS results for each knee model using the reference strain method where equation (4) is used to find the zero-load length. The RMS errors for simulation 12 are much lower than those found for the reference strain method for all three knees.

Analysis of variance (ANOVA) showed that both the zero-load length percentage for the ACL/PCL and LCL/MCL were significant factors in the kinematics for Knee \#1 $(\mathrm{P}<0.05)$ and for Knee \#3 $(\mathrm{P}=0.00)$. For Knee \#2 kinematics, only the zero-load length percentage for the LCL/MCL was significant $(\mathrm{P}<0.05)$.

\section{DISCUSSION}

The goals of this study were to: 1) validate a method for determining the subject specific zero-load length used in one-dimensional discrete element ligament models of the knee, 2) determine values for the zero-load length percentage parameter used in this method, 3) find the statistical significance of ligament model parameters to knee kinematics, and 4) compare the kinematic results of subject specific zero-load length parameters to the reference strain method which uses generic reference lengths found in literature.

In summary, we were able to validate this ligament modeling approach on three separate cadaver knees. We 
Table 5. The Average Orientation (Body 1, 2, 3) RMS Errors and the Normalized Orientation Errors for the Tibia Coordinate System Relative to the Femoral Coordinate System for Each Knee During each Simulation. Normalized Error Values for each Simulation were Added for all Three Knees. Note: The Top three Values in Each Column are Shown in Bold

\begin{tabular}{|c|c|c|c|c|c|c|c|}
\hline \multirow[t]{2}{*}{ Tibia 123} & \multicolumn{2}{|c|}{ Knee \#1 } & \multicolumn{2}{|c|}{ Knee \#2 } & \multicolumn{2}{|c|}{ Knee \#3 } & \multirow[t]{2}{*}{ NORM SUM } \\
\hline & RMS & NORM & RMS & NORM & RMS & NORM & \\
\hline 1 & 5.43 & 0.865 & 4.99 & 0.291 & N/A & N/A & N/A \\
\hline 2 & 5.26 & 0.780 & 4.42 & 0.193 & 19.03 & 1 & 1.97 \\
\hline 3 & 4.99 & 0.645 & 3.86 & 0.098 & 7.84 & 0.320 & 1.06 \\
\hline 4 & 3.98 & 0.134 & 3.96 & 0.115 & 3.32 & 0.045 & 0.294 \\
\hline 6 & 4.33 & 0.310 & 3.53 & 0.041 & N/A & N/A & N/A \\
\hline 7 & 4.31 & 0.299 & 9.10 & 1 & 16.70 & 0.858 & 2.16 \\
\hline 8 & 4.41 & 0.351 & 3.34 & 0.007 & N/A & N/A & N/A \\
\hline 9 & 4.43 & 0.364 & 5.81 & 0.433 & 14.37 & 0.717 & 1.51 \\
\hline 13 & 5.36 & 0.830 & 5.77 & 0.426 & 6.54 & 0.241 & 1.50 \\
\hline 14 & 4.11 & 0.201 & 3.45 & 0.027 & 3.93 & 0.082 & 0.310 \\
\hline 15 & 4.99 & 0.645 & 4.54 & 0.214 & 12.08 & 0.578 & 1.44 \\
\hline
\end{tabular}

Table 6. The Translation (x,y,z) and Orientation (Body 1, 2, 3) RMS Errors of the Tibia Coordinate System Relative to the Femoral Coordinate System for Each Knee During Simulation (12) ACL/PCL 85\% and LCL/MCL $75 \%$ and During the Simulation where the Reference Strain was Used to Find the Zero-Load Length. Translation Errors are in Millimeters and Orientation Errors are in Degrees. Note: Knee \#3 Failed Due to the Femur Slipping off of the Tibial Plateau in the Reference Strain Method

\begin{tabular}{|c|c|c|c|c|c|c|c|c|c|c|c|c|}
\hline & \multicolumn{6}{|c|}{ (12) ACL/PCL $85 \%$ \& LCL/MCL $75 \%$} & \multicolumn{6}{|c|}{ Reference Strain Method } \\
\hline & \multicolumn{3}{|c|}{ Translation (mm) } & \multicolumn{3}{|c|}{ Orientation $\left(^{\circ}\right)$} & \multicolumn{3}{|c|}{ Translation (mm) } & \multicolumn{3}{|c|}{ Orientation $\left({ }^{\circ}\right)$} \\
\hline & $\mathbf{x}$ & $\mathbf{y}$ & $\mathbf{z}$ & 1 & 2 & 3 & $\mathbf{x}$ & $\mathbf{y}$ & $\mathbf{z}$ & 1 & 2 & 3 \\
\hline$\# 1$ & 9.599 & 5.506 & 8.564 & 3.113 & 2.071 & 5.955 & 11.025 & 13.479 & 19.710 & 7.332 & 3.186 & 7.458 \\
\hline$\# 2$ & 5.596 & 4.416 & 2.862 & 1.762 & 0.995 & 7.126 & 16.704 & 8.412 & 6.093 & 3.566 & 4.098 & 17.114 \\
\hline \#3 & 10.125 & 4.370 & 2.087 & 1.545 & 1.979 & 6.094 & - & - & - & - & - & - \\
\hline
\end{tabular}

were also able to find values for the zero-load length percentage for the cruciates and collaterals that minimized kinematic error for all three cadaver knees. We also found that there is high statistical significant of this ligament modeling parameter to knee kinematics which validates the need for investigating this ligament modeling property. And finally, we found that for all three knees this subject specific method had much better kinematic results than using the generic reference strain method.

In this study it was found that for the Tibia RMS errors, the best simulations were 4,12 , and 14 . The overall best parameters were the cruciates (ACL and PCL) at $85 \%$ zeroload length and the collaterals (LCL and MCL) at $75 \%$ zeroload length (simulation 12). The next best simulation was with the cruciates at $85 \%$ and the collaterals at $80 \%$ (simulation 14). Simulation 4 was a close third with both the cruciates and the collaterals at $80 \%$.
These results show that the cruciates should be kept at a higher zero-load length percentage than the collaterals. Specifically, the cruciates should stay at $85 \%$ and not go any lower (tighter) whereas the collaterals should stay at $75 \%$ or $80 \%$ and not go any higher (looser). This could possibly be because the collaterals have much lower stiffness values $(\mathrm{k})$ than the cruciates. When the kinematic envelope of motion test is performed, the full range of motion of the knee joint is obtained while using minimal force. Since the collaterals have lower stiffness values, it takes much less force to stretch them than the cruciates. This means that there was a greater possibility that during the envelope of motion testing the collaterals were stretched more than the cruciates resulting in a larger than actual envelope of motion reading. In addition, when the envelope of motion test was performed there was a moment arm applied across the knee joint. This results in larger forces applied in the directions held by the collaterals than by the cruciates. It has also been found that 

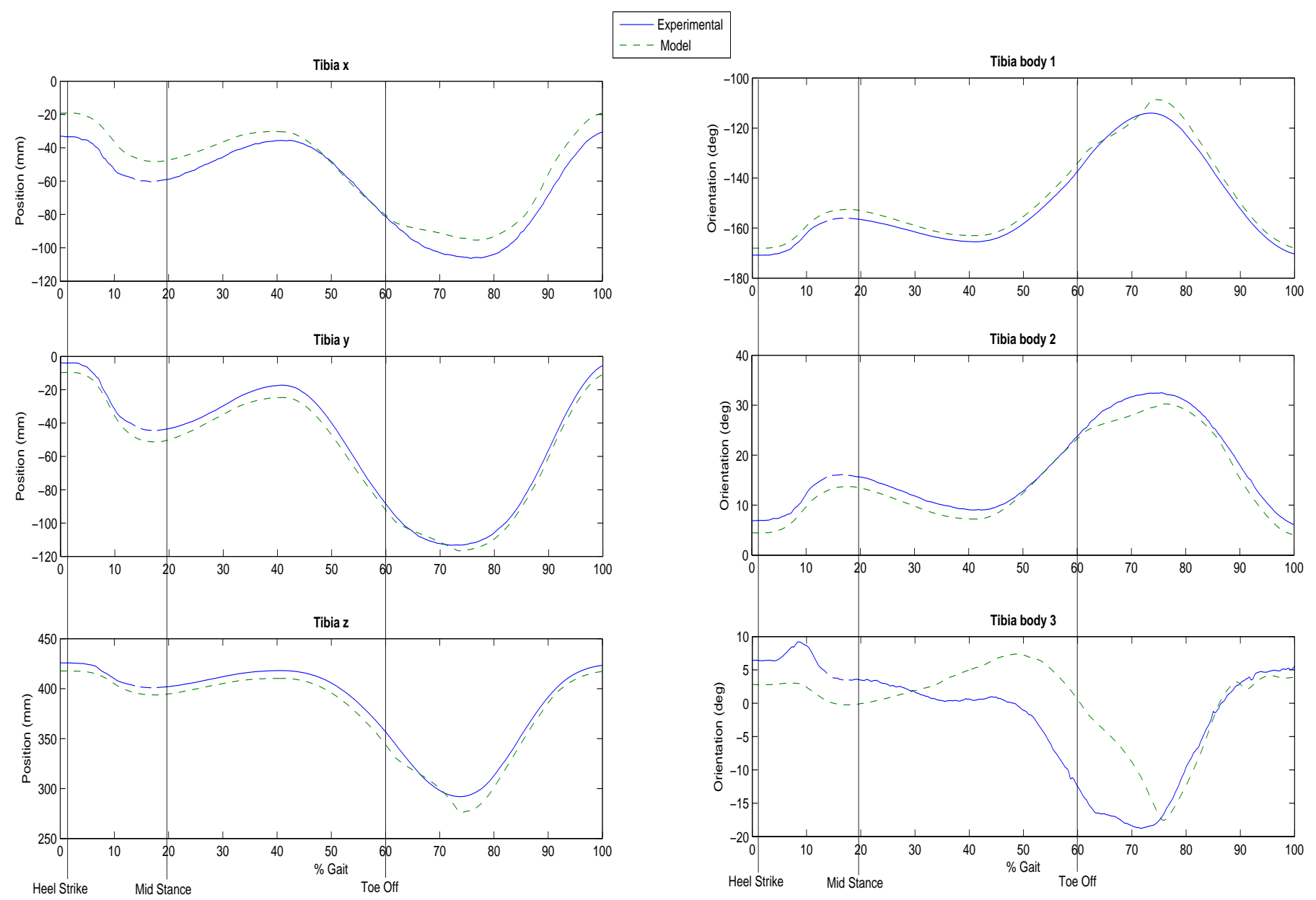

Fig. (3). Position $(x, y, z)$ and orientation (Body 1, 2, 3) of the tibia coordinate system relative to the femoral coordinate system for one walk cycle. This is comparing experimental data to model results for Knee \#1 during simulation (12) ACL/PCL 85\% and LCL/MCL 75\%.

the two bundles of the ACL and PCL twist around each other during flexion $[20,25]$ whereas although the bundles in the LCL and MCL wrap around bone, they do not wrap around each other. The twisting of the cruciates could cause the bundles to become stiffer during the envelope of motion testing than the collaterals.

Both of the zero load length parameters proved to be significant factors in the analysis of variance testing (with $\alpha$ $=0.05$ ). For tibia translation, both ACL/PCL and LCL/MCL percentage was significant for Knee \#1 and Knee \#3. In addition, LCL/MCL percentage was a significant factor for all three knees for the tibia orientation. Overall, these results prove that the knee kinematics during the simulated walk cycle were extremely sensitive to the zero-load length parameters of both the cruciates and the collaterals. Similarly, it has been found previously by Bertozzi et al. [14] that laxity in the knee is extremely sensitive to variations in the reference length parameter. In addition, Baldwin et al. [4] found that the reference strain parameter was a highly important factor in their sensitivity study (as noted before, the reference length and reference strain is related to the zero-load length parameter by equation 4 ).

These results not only verify the importance of the zeroload length value when modeling the knee joint but also verify that doing the manual envelope of motion test is a valid approach to find the passive range of motion of the knee joint. Table 6 compares the RMS error results for simulation 12 versus using the reference strain method. The RMS errors are much higher when the reference strain method was used as compared to using the experimentally found subject specific zero-load lengths. In addition, the walk cycle using the reference strain method with Knee \#3 completely failed due to the femur slipping off of the tibial plateau. This finding is also backed up in the literature, it has been found that the passive limits of the knee joint can be easily found by hand manipulation if it is done properly [3033]. This study has shown that there must be a correction percentage used when calculating the zero-load length of the ligaments using this kinematic envelope of motion method most likely due to the fact that when in the non-linear toe region, the ligaments stretch under very minimal force application. This method has shown an approach to directly find the zero-load length of each ligament that can be applied to three different cadaver knees. In the past, knee models have used the reference strain method which uses the reference length and reference strain to then back out the zero-load length. If done properly (and scaled using the percentages found in this study), this method is a much more direct approach to modeling the ligament force-displacement behavior. 


\section{Study Limitations}

It should be noted that there were some limitations in this study. To begin with, ligament wrapping was not included in any of the three knee models. Adding this would most likely improve the overall RMS error results for all of the knee models, so it could slightly change the sensitivity parameter results found in this study. In addition, the menisci were not included in any of the three knee models. The menisci are important load-bearing structures in the knee and previous work has been done in modeling the menisci in these multibody knee models [8]. The results showed that the menisci have a significant effect on the distribution of tibiofemoral contact forces, but have a statistically insignificant effect on tibio-femoral kinematics during the walk cycle simulated in the knee simulator [8]. Since the present study focused on the relative kinematics of the tibio-femoral joint, the absence of the menisci should not significantly influence the kinematic results presented. In addition, only a single walk cycle simulation was investigated. Since we know that the ligaments undergo much higher stresses in other motions it would be beneficial to expand this study to a broad range of knee motions.

It should also be noted that errors in the tibia body 3 orientation were slightly larger than the other position and orientation errors as can be seen in Fig. (3) and Table 6. Much of this error can be attributed to the model representing the dynamic knee simulator. The model has the largest errors in the medial-lateral direction of the ankle sled which manifests in the tibia body 3 rotation measurements. The increased error in the tibia body 3 rotation has been previously documented when modeling an instrumented analogue knee and prosthetic knees [34] as well as in natural knee models. These errors were consistent among the models presented in this study and should not affect the relative kinematic results.

\section{Future Work}

The ligaments in each of the three knee models for this study were all non-linear line springs elements. Ligament wrapping was not modeled, but this is something that is currently being worked on, especially for the cruciates which are known to not only wrap around bone, but also around other ligament bundles. Another aspect that could be added to the models is to have the ligament insertion and origin sites span the entire area and not just be attached at a single point. Each of these improvements to the model should improve our understanding of how the ligaments are actually strained during everyday normal activities.

Weiss et al. [2] noted that this sort of research will "provide the means to apply subject-specific modeling techniques to the study of the joints of individual patients." In the future we would like to apply our knee modeling techniques in vivo to human subjects (not only cadaver knees). We would like to create and simulate living subjectspecific knee and lower limb models. This way we will be able to use the gait lab to take actual movement data and then apply that to knee models for human subjects. The kinematic envelope of motion test is something that can be done on living subjects to find their specific zero-load lengths. It would also be beneficial to compare ligament properties for living subjects versus cadaver knees. There is a chance that different zero-load length percentages need to be used for living subjects, this is something that can be tested in the future.

\section{CONFLICT OF INTEREST}

There are no conflicts of interest.

\section{ACKNOWLEDGMENTS}

This research was funded by the National Science Foundation, Grant Number 506297, under the IMAG program for Multiscale Modeling. The authors would also like to acknowledge the work of Mohammad Kia, Leo Olcott, and Hongzeng Liu for their work on this project in the Musculoskeletal Biomechanics Research Lab at UMKC.

\section{REFERENCES}

[1] L. Bertozzi, R. Stagni, S. Fantozzi, and A. Cappello, "Knee model sensitivity to cruciate ligaments parameters: A stability simulation study for a living subject", J. Biomech, vol. 40, pp. 8-44, 2007.

[2] J. A. Weiss, J. C. Gardiner, B. J. Ellis, T. J. Lujan, and N.S. Phatak, "Three-dimensional finite element modeling of ligaments: Technical aspects", Med. Eng. Phys., vol. 27, pp. 845-861, 2005.

[3] J. A. Weiss and J. C. Gardiner, "Computational Modeling of Ligament Mechanics", Crit. Rev. 'Biomed. Eng., vol. 29, no. 4, pp. $1-70,2001$.

[4] M. A. Baldwin, P. J. Laz, J. Q. Stowe, and P. J. Rullkoetter, "Efficient probabilistic representation of tibiofemoral soft tissue constraint", Comp. Meth. Biomech. Biomed. Eng., vol. 12, no. 6, pp. 651-659, 2009.

[5] L. Blankevoort, J. H. Kuiper, R. Huiskes, and H. J. Grootenboer, "Articular Contact in a three-dimensional model of the knee", $J$. Biomech, vol. 24, no. 11, pp. 1019-1031, 1991.

[6] L. Blankevoort and R. Huiskes, "Ligament-Bone Interaction in a three-dimensional model of the knee", J. Biomech Eng., vol. 113, pp. 263-269, 1991 .

[7] L. Blankevoort and R. Huiskes, "Validation of a three-Dimensional model of the knee", J. Biomech, vol. 26, pp. 955-961, 1996.

[8] T. M. Guess, G. Thiagaragan, M. Kia, and M. Mishra, "A subject specific model of the knee with menisci", Med. Eng. Phys., vol. 32, pp. 505-515, 2010.

[9] G. Li, J. Gil, A. Kanamori, and S. L. Y. Woo, "A validated threedimensional computational model of a human knee joint", $J$. Biomech. Eng., vol. 121, pp. 657-662, 1999.

[10] K. Weimer, "Development and Validation of a Subject-Specific Computational Human Knee Model in a Dynamic Knee Simulator to Include Ligament and Tendon Bone Wrapping", M.S. thesis, University of Missouri - Kansas City, Kansas City, MO, 2007.

[11] J. Wismans, F. Veldpaus, J. Janssen, A. Huson, and P. Struben, "A Three-Dimensional Mathematical Model of the Knee-Joint”, J. Biomechanics, vol. 13, no. 8, pp. 677-685, 1980.

[12] N. H. Yang, P. K. Canavan, H. Nayeb-Hashemi, B. Najafi, and A. Vaziri, "Protocol for constructing subject-specific biomechanical models of knee joint", Comp. Methods Biomech. Biomed. Eng., vol. 13, no. 5, pp. 589-603, 2010.

[13] G. Li, T. J. Gill, L. E. DeFrate, S. Zayontz, V. Glatt, and B. Zarins, "Biomechanical consequences of PCL deficiency in the knee under simulated muscle loads - an in vitro experimental study", J. Orthopaedic Res., vol. 20, no. 4, pp. 887-892, 2002.

[14] L. Bertozzi, R. Stagni, S. Fantozzi, and A. Cappello, "Evaluation of a cruciate ligament model: sensitivity to the parameters during drawer test simulation", J. Appl. Biomech, vol. 24, pp. 234-243, 2008.

[15] L. P. Maletsky and B. M. Hillberry, "Simulating dynamic activities using a five-axis knee simulator", J. Biomech. Eng., vol. 127, no. 1, pp. 123-133, 2005.

[16] T. M. Guess and L. P. Maletsky, "Computational Modeling of a Dynamic Knee Simulator for Reproduction of Knee Loading", J. Biomech. Eng., vol. 127, no. 7, pp. 1216-1221, 2005.

[17] ISO 14243-1, "Implants for surgery - Wear of total knee-joint prostheses - Part 1: Loading and displacement parameters for weartesting machines with load control and corresponding environmental conditions for test", 2002. 
[18] L. P. Maletsky, J. Sun, and N. A. Morton, "Accuracy of an optical active-marker system to track the relative motion of rigid bodies", J. Biomechanics, vol. 40, no. 3, pp. 682-685, 2007.

[19] S. J. Piazza and S. L. Delp, "Three-Dimensional dynamic simulation of total knee replacement motion during a step-up task", J. Biomech. Eng., vol. 123, no. 6, pp. 599-606, 2001.

[20] L. E. DeFrate, T. J. Gill, and G. Li, "In vivo Function of the posterior cruciate ligament during weightbearing knee flexion", Am. J. Sports Med., vol. 32, no. 8, pp. 1923-1928, 2004.

[21] A. Chhabra, J. S. Starman, M. Ferretti, A. F. Vidal, T. Zantop, and F. H. Fu, "anatomic, radiographic, biomechanical, and kinematic evaluation of the anterior cruciate ligament and its two functional bundles", J. Bone Joint Surg., vol. 88, suppl. 4, pp. 2-10, 2006.

[22] S. E. Park, L. E. DeFrate, J. F. Suggs, T. J. Gill, H. E. Rubash, and G. Li, Erratum to "The change in length of the medial collateral ligaments during in vivo knee flexion", Knee, vol. 13, pp. 77-82, 2006.

[23] D. L. Butler, M. D. Kay, and D. C. Stouffer, "Comparison of material properties in fascicle-bone units from human patellar tendon and knee ligaments", J. Biomech., vol. 19, pp. 425-432, 1986.

[24] S. E. Park, L. E. DeFrate, J. F. Suggs, T. J. Gill, H. E. Rubash, and G. Li, "The change in length of the medial and lateral collateral ligaments during in vivo knee flexion", Knee, vol. 12, pp. 377-382, 2005.

[25] V. B. Duthon, C. Barea, S. Abrassart, J. H. Fasel, D. Fritschy, and J. Menetrey, "Anatomy of the anterior cruciate ligament", Knee Surg. Sports Traumatol. Arthrosc., vol. 14, pp. 204-213, 2006.

[26] S. S. Jordan, L. E. DeFrate, K. W. Nha, R. Papannagari, T. J. Gill, and G. Li, "The in vivo kinematics of the anteromedial and posterolateral bundles of the anterior cruciate ligament during weightbearing knee flexion", Am. J. Sports Med., vol. 35, pp. 547554, 2007.

[27] R. Papannagari, L. E. DeFrate, K. W. Nha, J. M. Moses, M. Moussa, T. J. Gill, and G. Li, "Function of posterior cruciate ligament bundles during in vivo knee flexion", Am. J. Sports Med., vol. 35, pp. 1507-1512, 2007.

[28] W. Petersen and T. Zantop, "Anatomy of the anterior cruciate ligament with regard to its two bundles", Clin. Orthop. Relat. Res., vol. 454, pp. 35-47, 2007.

[29] N. A. Morton, L. P. Maletsky, S. Pal, and P. J. Laz, "Effect of variability in anatomical landmark location on knee kinematic description", J. Orthop. Res., vol. 25, pp. 1221-1230, 2007.

[30] K. Dodd, 'knee's motion path relative to the passive coupled kinematic envelope", M.S. thesis, University of Kansas, Lawrence, KS, 2009.

[31] S. Nielsen, "Kinesiology of the knee joint. An experimental investigation of the ligamentous and capsular restraints preventing knee instability", Dan. Med. Bull., vol. 34, no. 6, pp. 297-309, 1987.

[32] L. Blankevoort, R. Huiskes, and A. de Lange, "The envelope of passive knee joint motion", J. Biomech., vol. 21, no. 9, pp. 705$720,1988$.

[33] J. A. Sidles, R. V. Larson, J. L. Garbini, D. J. Downey, and F. A. Matsen, "Ligament length relationships in the moving knee", $J$. Orthop. Res., vol. 6, no. 4, pp. 593-610, 1988.

[34] T. M. Guess, "Computational modeling of a dynamic knee simulator", Ph.D. thesis, University of Kansas, Lawrence, KS, 2003.

Received: January 01, $2012 \quad$ Revised: February 25, 2012

(C) Bloemker et al.; Licensee Bentham Open.

This is an open access article licensed under the terms of the Creative Commons Attribution Non-Commercial License (http://creativecommons.org/licenses/by-nc/3.0/) which permits unrestricted, non-commercial use, distribution and reproduction in any medium, provided the work is properly cited. 